\title{
Analysis of some specular nano-oxide-layer spin valve used for digital applications
}

\author{
Daniela Ionescu*, and Petre-Daniel Matasaru
}

Gheorghe Asachi Technical University of Iasi, Department of Telecommunications, Carol I Blvd., No.11, 700506 Iasi, Romania

\begin{abstract}
Some peculiar spin valve structures with increased magneticresistance (MR), based on Co alloys, have been analyzed in this paper by simulation methods (with help of the HFSS 13.0 program) and their performances were determined for applications at digital sensors. Structures involve specular nano-oxide-layer spin valves (NOL-SV) presenting a NOL insertion inside the pinned layer. A controlled structure has been obtained, simulations indicating the parameters correlation in order to obtain the MR maxima. Values of around $5.8 \ldots 16 \%$ for the MR ratio have been obtained, respectively a variation of the resistance-area product of $0.7 \ldots 9 \mathrm{~m} \Omega \cdot \mu \mathrm{m}^{2}$, for an individual layer thickness of around 2-3.2 nm, in an applied field of about $0.4 \ldots 2.7 \mathrm{kOe}$. Our results illustrate better performances of the NOL spin valves in comparison with the results previously reported in literature.
\end{abstract}

\section{Introduction}

In a spin valve, two magnetic layers separated by a nano metric spacer present electrical resistance which can change between two values depending on the relative alignment of the magnetization in the layers. The resistance change is a result of the giant magnetoresistive (GMR) effect in the structure.

In the specular nano-oxide-layer spin valves (NOL-SV), nano-oxide layers are introduced inside the layer with pinned magnetization and above the layer with free orientable magnetization in order to obtain a specular scattering effect of the spin-polarized electrons, which has as a result the increasing of the magnetic resistance ratio (MR) of the structure, for thinner sense layers. Geometrical dimensions and physical nature of the layers in the spin valve influence significantly the magnetoresistive effect inside the material samples and their fine variation can determine resonant evolution of the MR in some specific domains [12], [14].

This type of spin valves are used in read head and related digital applications. Among these one can mention electric and electronic devices like: magnetic read heads, magnetic field sensors and GMR isolators, microelectromechanical systems (MEMS) and other devices [1], [3], [13], [15]. The magnetic field sensors are devices used to read data in hard disk drives, or like biosensors, etc.

\footnotetext{
*Corresponding author: danaity@yahoo.com
} 


\section{Spin valves characterization}

\subsection{Theoretical considerations}

The configuration of the stack of layers in the NOL spin valve was represented in Figure 1. The main magnetic layers (with pinned, respectively free magnetization) are separated by a nanometric non-magnetic spacer. The theoretical support of the oscillatory antiferromagnetic coupling of the magnetic layers in the stack was considered: the RKKY (Ruderman - Kittel - Kasuya - Yosida) theory and the quantum well models. The intensity of the antiferromagnetic coupling depends strongly on the nonmagnetic spacer properties and in the case of the NOL-SV, on the NOL layers positions in the stack.

For estimating the MR of the considered spin valves using the simulation data, the following formula has been applied, using the sensing current which transforms the change in resistance in a readback voltage [1], [2], [12], given by simulation:

$$
\frac{d R}{R}=\frac{R(H)-R(0)}{R(0)}
$$

Where $R(H)$ is the resistance of the sample in a magnetic field $H$, and $R(0)$ is the resistance in null field.

The current-perpendicular-to-plane (CPP) geometry was considered, where the sensing current flows perpendicular to the ferro / nonferro-magnetic interfaces. The specific resistance, $A R$ represents the product of the area $A$ through which an uniform CPP current flows, and the sample resistance $R$. Variation of this parameter is:

$$
\Delta A R=A R_{\text {antill }}-A R_{\mathrm{II}}
$$

Which represents the difference between the specific resistances in the anti-parallel and parallel states (magnetizations of adjacent ferromagnetic layers in the stack) [4], [5], [10], [11]. On the context, the CPP magnetic resistance ratio was considered as [14], [15], [16]:

$$
M R_{\mathrm{CPP}}[\%]=\frac{\Delta A R}{A R_{\text {antill }}}
$$

\subsection{NOL spin valves structure}

Some peculiar spin valve structures with increased magnetic resistance (MR), based on Co alloys, have been analyzed in this paper by simulation methods (with help of the HFSS 13.0 program) and their performances were determined. Structures involve specular nano-oxidelayer spin valves (NOL-SV), presenting a NOL insertion inside the pinned layer and also above the free layer. Thickness of the oxidation layer is about $1 \mathrm{~nm}(0.5-2 \mathrm{~nm}$ for parametrical analysis).

The NOL-SV structure of layers (from bottom to above layers) is represented in Fig 1: $\mathrm{Ta}(3.7 \mathrm{~nm}) / \mathrm{NiFeCr}(2.4 \mathrm{~nm}) / \mathrm{PtMn}(5.5 \mathrm{~nm}) / \mathrm{CoCr}_{15} \mathrm{Pt}_{12}$ (pinned, $\left.1.8 \mathrm{~nm}\right) / /$ oxidation (NOL1) // $\mathrm{CoCr}_{15} \mathrm{Pt}_{12}$ (pinned, $2.2 \mathrm{~nm}$ ) / Ru (2 nm) (spacer) / $\mathrm{CoCr}_{15} \mathrm{Pt}_{12}$ (free, $2.8 \mathrm{~nm}$ )// oxidation (NOL2, $\mathrm{Ta}_{2} \mathrm{O}_{5}$ ) // Ta $(3.5 \mathrm{~nm}$ ) capping layer. Structure is placed on Si substrates.

Above a buffer layer (Ta or Ti) and an underlayer, the antiferromagnetic (AFM) PtMn layer is placed. This AFM layer represents a pinning layer, which fixes the magnetization of the bottom hard magnetic layer and raises its coercivity. The hard magnetic $\mathrm{CoCr}_{15} \mathrm{Pt}_{12}$ layer 
(in contact with the AFM PtMn layer) represents the pinned layer. It is divided in two parts, inside it a nano-oxide-layer (NOL1) is grown in an oxidation process. After a nanaometric non-magnetic spacer, a magnetic layer with free orientable magnetization is placed: the $\mathrm{CoCr}_{15} \mathrm{Pt}_{12}$ layer. The capping layer encloses the structure, below which an oxidation layer NOL2 $\left(\mathrm{Ta}_{2} \mathrm{O}_{5}\right)$ is grown.

The hard magnetic material was considered: $\mathrm{CoCr}_{15} \mathrm{Pt}_{12}-\mathrm{CoCr}_{22} \mathrm{Pt}_{14}$, respectively CoCrTa. For the oxide of the pinned layer we have considered the possibilities reported in literature [2], [6], [8]: in NOL1: $\mathrm{CoO}, \mathrm{CrO}, \mathrm{CoCr}_{2} \mathrm{O}_{4}, \mathrm{Cr}_{2} \mathrm{O}_{3}, \mathrm{Cr}_{3} \mathrm{O}_{4}$, respectively $\mathrm{Co}_{3} \mathrm{O}_{4}$.

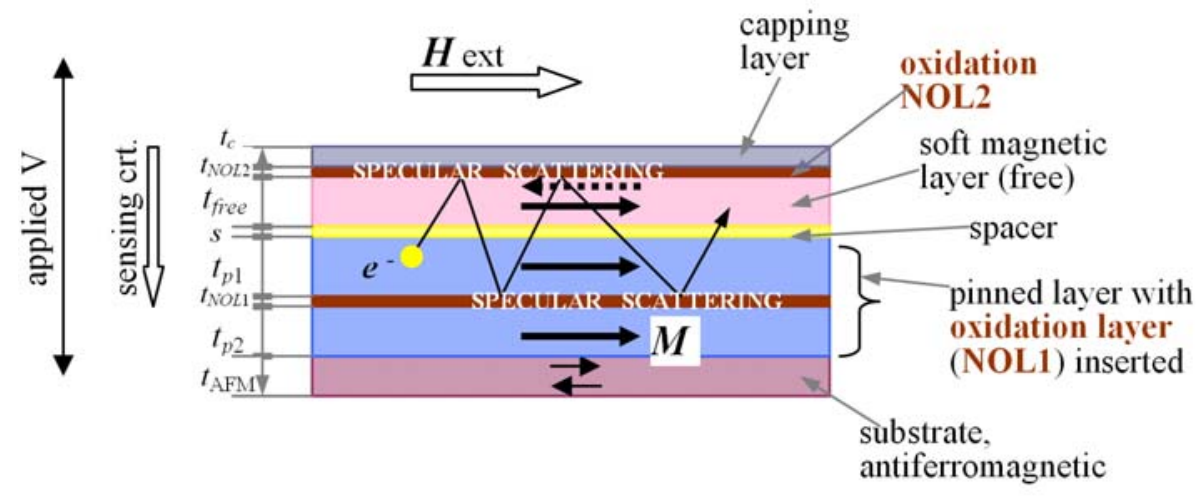

Fig. 1. Layer configuration of the considered specular nano-oxide-layer spin valve. The currentperpendicular-to-plane (CPP) geometry was considered, where the sensing current flows perpendicular to the ferro / nonferro-magnetic interfaces.

In the specular nano-oxide-layer spin valve, the sensing current flows perpendicular to the ferro / nonferro-magnetic interfaces, in the considered current-perpendicular-to-plane (CPP) geometry (Figure 1).

The role of the two nano-oxide-layers in SV is to enhance the specular reflection of spinpolarized electrons from the oxide interface, while conserving their spin direction [4], [5]. The above oxidation layer NOL1 allows the direct exchange coupling between the PtMn layer and the pinned CoCrPt layer. The mean free path of majority spin-polarized electrons is extended, due to specular reflection at metal and insulator interfaces. In the same time, the coercitive field $H_{c}$ increases when an oxidation layer is inserted into the pinned layer [5], [8], [9].

The main effect is that the GMR ratio is enhanced by insertion of the oxidation layers inside the pinned layer and after the free magnetic layer [4], [8].

\section{Results for structure magnetic resistance}

We have used the structural simulation data to calculate the CPP magnetic resistance ratio for the considered spin valve structures, presenting oxidation layers. The individual layer thickness in the stack in Fig. 1 was around 1.8...5.5 nm, depending of the layer, in an applied field of about $0.4 \ldots 2.7 \mathrm{kOe}$. A sense current, $I$, of $0 \ldots 3 \mathrm{~mA}$ has been considered in the stack of layers. For parametrical representation, the NOL1 layer thickness $t_{\mathrm{NOL} 1}$ was about $0.5 \ldots 2$ $\mathrm{nm}$, while the free layer thickness $t_{\text {free }}$ was of $1.8 \ldots 3.5 \mathrm{~nm}$.

For the 3D structural simulations, the mesh was set in the ANSYS HFSS program (High Frequency Electromagnetic Field Simulator) at $16.6 \times 16.6 \times 22.4 \mathrm{~nm}^{3}$ per cell, chosen to overall the exchange interaction length for the magnetic layers in the stack, which is of nanometers order [13], [15], [19].

The resistance-area product and it's variation was also calculated for the NOL-SV with the two kind of hard magnetic material ( $\mathrm{CoCrPt}$, respectively $\mathrm{CoCrTa})$. 
Graphs for the MR ratio as a function of the free layer thickness $t_{f r e e}$ are given in Fig. 2, with the NOL1 layer thickness $t_{\mathrm{NOL} 1}$ as parameter. The CPP magnetic resistance ratio presents an increasing with $t_{\text {free }}$, with a wide maximum which occurs around $2.9 \mathrm{~nm}$ for $t_{\text {free }}$, value imposed by the magnetic ion nature in the hard magnetic alloy.

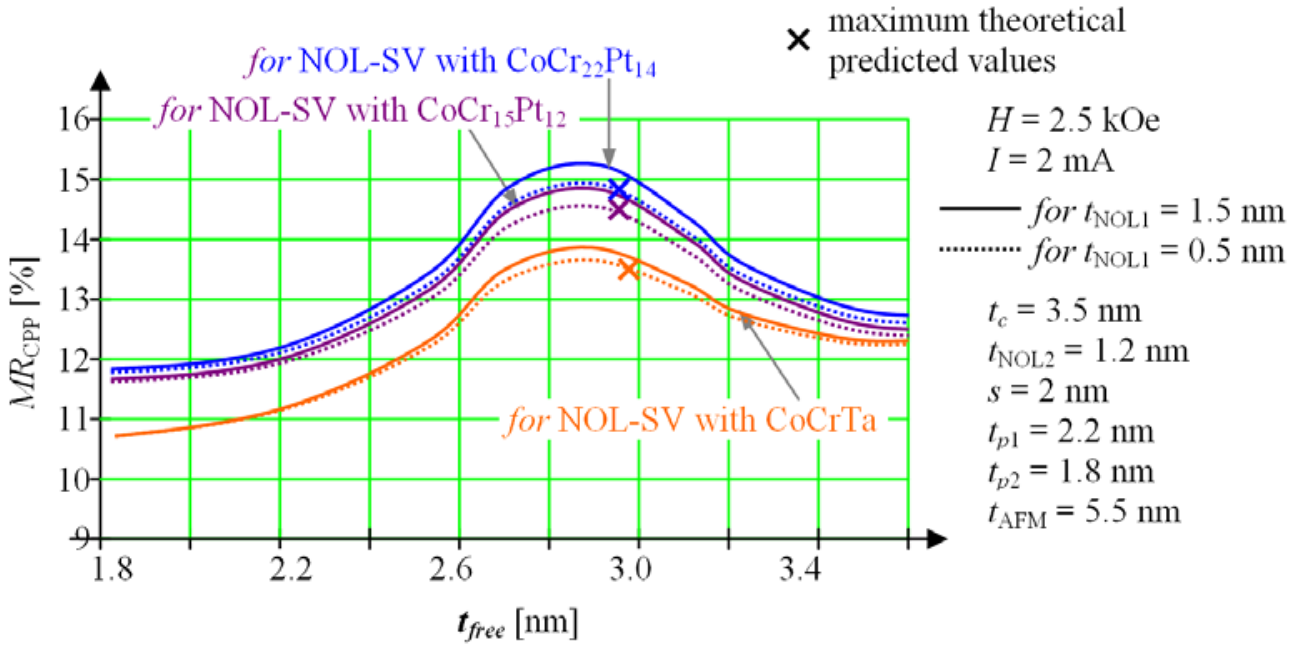

Fig. 2. The CPP magnetic resistance ratio of the considered specular nano-oxide-layer spin valves as a function of the free layer thickness $t_{\text {free. }}$. Theoretical predicted maxima were represented on graphs.

One can observe that higher content of $\mathrm{Cr}$ in the hard magnetic alloy determines higher values for magnetic resistance. Concerning the materials conductivity, the Pt conductivity of $0.96 \cdot 10^{7} \Omega^{-1} \cdot \mathrm{m}^{-1}$ being lower in comparison with Ta conductivity of $0.76 \cdot 10^{7} \Omega^{-1} \cdot \mathrm{m}^{-1}$, generates a little bit fewer conduction electrons participating at scattering processes, which determines lower values of the magnetic resistance.

Theoretical predicted maximum on each graph occurs for thicker free magnetic layer (corresponding to a higher magnetization value in the layer) and displays a lower value. The oxidation layer thickness influences the curves, thicker oxidation layer being equivalent with a more accentuate specular scattering effect and avoiding the possible tunneling phenomena.

The distance effect is also reported in literature, the GMR effect increasing generally versus the thickness of individual magnetic layers, but the curves shape depends on the distance of the NOL to the magnetic layers [4], [7], [8].

Graphs for the variation of the resistance-area product as a function of spacer thickness $s$ were given in Figure 3.

The thickness of above part of the hard magnetic layer $t_{p 1}$, which represents also the distance in this layer to the below oxidation layer NOL1, was considered as parameter. The free layer thickness and thickness of the NOL1 layer were considered for obtaining the maximum GMR effect in the spin valve. 


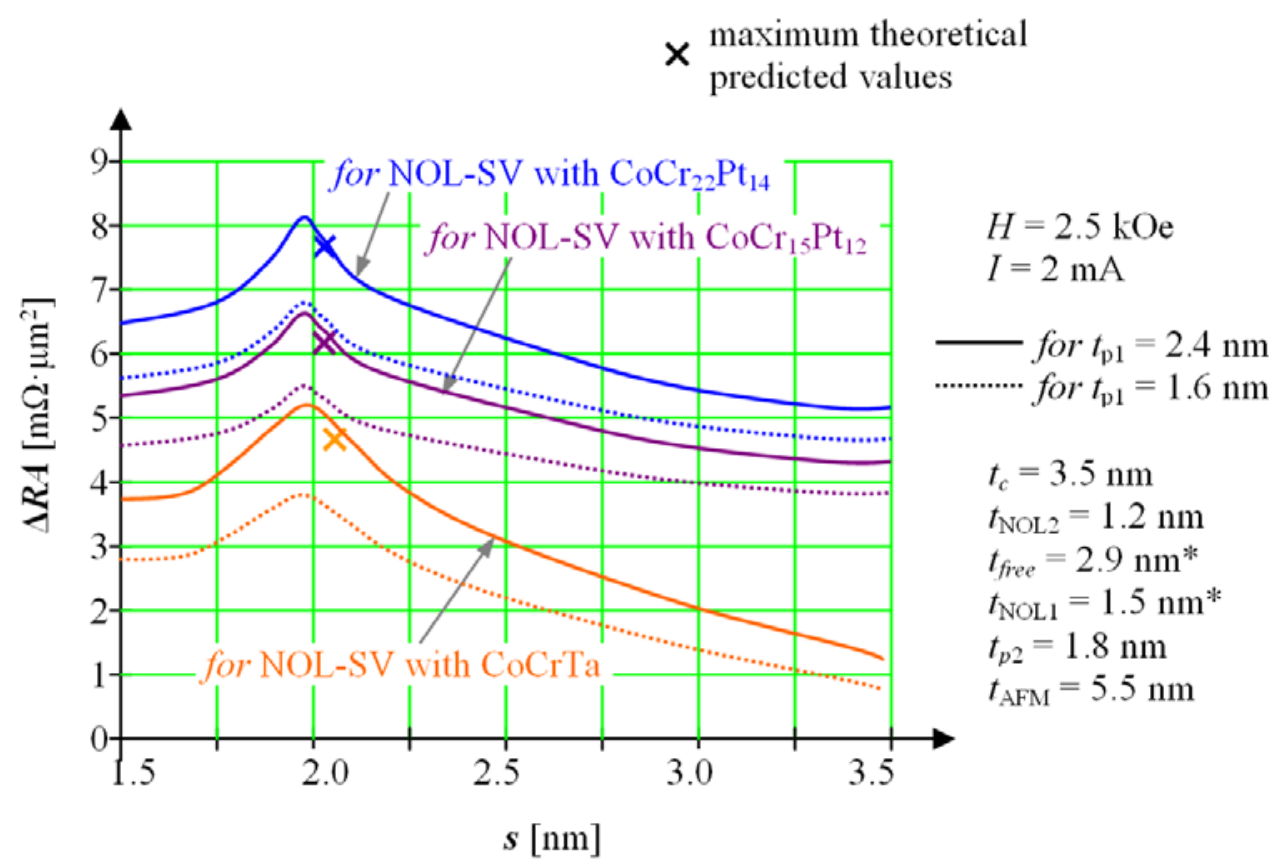

Fig. 3. Variation of the resistance-area product as a function of spacer thickness $s$, for the considered specular nano-oxide-layer spin valves. Theoretical predicted maxima were represented on graphs.

A variation of the resistance-area product of $0.7 \ldots 9 \mathrm{~m} \Omega \bullet \mu \mathrm{m}^{2}$ was obtained, presenting a maximum around a value for spacer thickness of $2 \mathrm{~nm}$. Even the $R A$ values are decreasing when the nano-oxide-layers are inserted in the spin valve, the occurring maximum is of interest, implying higher values of the CPP magnetic resistance ratio (rel (1)), while the antiparallel specific resistance $A R_{a n t i \|}$ also decreases.

\section{Conclusions}

Structures involve specular nano-oxide-layer spin valves (NOL-SV) presenting a NOL insertion inside the pinned layer have been analyzed in this paper. A controlled structure has been obtained, simulations indicating the parameters correlation in order to obtain the MR maxima.

Values of around $5.8 \ldots 16 \%$ for the MR ratio have been obtained, respectively a variation of the resistance-area product of $0.7 \ldots 9 \mathrm{~m} \Omega \bullet \mu \mathrm{m} 2$, for an individual layer thicknesses of around $1.8 \ldots 5.5 \mathrm{~nm}$, in an applied field of about $0.4 \ldots 2.7 \mathrm{kOe}$.

Our results illustrate better performances of the NOL spin valves in comparison with the results previously reported in literature for the classic spin valves [13], [17], [18], [19].

The GMR ratio is enhanced by insertion of the nano-oxide-layers inside the pinned layer and after the free layer, thus creating a specular scattering space for the spin-polarized electrons.

Geometrical dimensions (especially active layers and spacer thicknesses) and physical nature of the layers in the spin valve influence significantly the magnetic resistance variation at device level and the obtained resonances of the magnetic resistance ratio in some specific domains are of great importance for particular applications.

Performances of the NOL-SV imply two great improvements: a higher MR ratio and thinner sense-layer inside the spin valve structure. 


\section{References}

1. C. Reig ; D. Ramirez ; H.H. Li ; P.P. Freitas, IEE Proceedings - Circuits, Devices and Systems 152, 4, 5 (2005)

2. L. Wang, J. Qiu, W.J. McMahon, Y.H. Wu, Physical Review B 69, 21 (2004)

3. J.Dae Suh, C. A. Ross, Physica Status Solidi (B) 244, 12 (2007)

4. F. Shen, Q. Y. Xu, G. H. Yu, W. Y. Lai, Z. Zhang, Z. Q. Lu, G. Pan, A. Al-Jibouri, Applied Physics Letters 80, 23 (2002)

5. F. Shen, Q. Y. Xu, G. H. Yu, Z. Q. Lu, J.-W. Cai, Z. Zhang, Acta Physica Sinica 51, 8 (2002)

6. S. Y. Yoon, D. H. Lee, J. Y. Lee, Y. S. Kim, D. H. Yoon, S. J. Suh, Journal of the Korean Physical Society 44, 5 (2004)

7. C. Yongxiang, W. Haicheng, Y. U. Guanghua, Journal of University of Science and Technology Beijing 30, 12 (2008)

8. C.L. Platt, K.W. Wierman, E.B. Svedberg, T.J. Klemmera, J.K. Howarda, David J. Smith, Journal of Magnetism and Magnetic Materials 247, 2 (2002)

9. J. Ventura, J. Sousa, J. P. Araujo, P. P. Freitas, Journal of Applied Physics 101, (2007)

10. Y. Xu, D. D. Awschalom, J. Nitta, Junsaku (Eds.), Handbook of Spintronics (Springer Netherlands, 2016)

11. T. Dietl, D. Awschalom, M. Kaminska, H. Ohno (Eds.), Spintronics, Volume 82, 1st Edition, (Academic Press, Elsevier, 2008)

12. D.D. Awschalom, D. Loss, N. Samarth (Eds.), Semiconductor Spintronics and Quantum Computation (Springer Science \& Business Media, 2013)

13. B. Seongtae, H. Jack, Spin Valves in Spintronics Applications: Materials, Device Applications, and Electrical (VDM Verlag Dr. Müller, 2010)

14. K. Sato, E. Saitoh (Eds.), A. Willoughby, P. Capper, Safa Kasap (Series Eds.), Spintronics for Next Generation Innovative Devices, (Wiley, 2015)

15. G. C. Lombardi, G. E. Bianchi, Spintronics: Materials, Applications and Devices (Nova Science Publishers, 2009)

16. E. R. Hedin, Y. S. Joe (Eds.), Spintronics in Nanoscale Devices (CRC Press, 2016)

17. R. A. Lukaszew (Ed.), Handbook of Nanomagnetism, Applications and Tools, (Pan Stanford Publishing Pte. Ldt., Singapore, 2015)

18. H. Yu, L. Ni, Y. Wang, Non-Volatile In-Memory Computing by Spintronics (Synthesis Lectures on Emerging Engineering Technologies), (Morgan \& Claypool Publishers, 2016)

19. C. Felser, G. H. Fecher (Eds.), Spintronics: From Materials to Devices, (Springer Science \& Business Media, 2013) 